\title{
Adjustable Permanent Quadrupoles for the Next Linear Collider
}

\author{
J. T. Volk*, J. DiMarco, G.W. Foster, W. Fowler, V. S. Kashikhin, A. Makarov, V. Tsvetkov \\ Fermi National Accelerator Laboratory
}

\author{
C. E. Rago, A. Ringwall, C. M. Spencer, Z. Wolf \\ Stanford Linear Accelerator Center
}

\begin{abstract}
The proposed Next Linear Collider (NLC) will require over 1400 adjustable quadrupoles between the main linacs' accelerator structures. These $12.7 \mathrm{~mm}$ bore quadrupoles will have a range of integrated strength from 0.6 to 138 Tesla, with a maximum gradient of 141 Tesla per meter, an adjustment range of +0 to $-20 \%$ and effective lengths from $324 \mathrm{~mm}$ to $972 \mathrm{~mm}$. The magnetic center must remain stable to within 1 micron during the $20 \%$ adjustment. In an effort to reduce costs and increase reliability, several designs using hybrid permanent magnets have been developed. Four different prototypes have been built. All magnets have iron poles and use Samarium Cobalt to provide the magnetic fields. Two use rotating permanent magnetic material to vary the gradient, one uses a sliding shunt to vary the gradient and the fourth uses counter rotating magnets. Preliminary data on gradient strength, temperature stability, and magnetic center position stability are presented. These data are compared to an equivalent electromagnetic prototype.
\end{abstract}

\section{NLC DESIGN}

The Next Linear Collider ${ }^{1}$ (NLC) is future electron/positron collider that is based on copper accelerator structures powered with $11.4 \mathrm{GHz} \mathrm{X}$-band RF. It is designed to begin operations with a center-of-mass energy of $500 \mathrm{GeV}$ or less, depending on the physics interest, and to be adiabatically upgraded to $1 \mathrm{TeV} \mathrm{cms}$ with a luminosity of $2 \sim 3 \times 10^{34} \mathrm{~cm}^{-2} \mathrm{~s}^{-1}$. The facility is roughly $30 \mathrm{~km}$ in length and supports two independent interaction regions. For the main linac there will be over 1400 quadrupoles between the accelerator structures. To reduce costs and increase reliability adjustable permanent magnets are considered for these structures. Based on Fermilab's experience with permanent magnets used in their Recycler, a collaboration between SLAC and Fermilab is exploring designs and prototypes.

\section{MAGNET REQUIREMENTS}

The general linac magnet requirements are the same for

Submitted June 2001

*Volk@fnal.gov

Work supported by the U.S. Department of Energy under contract No. DE-AC02-76CH03000 and DE-AC03-

$76 \mathrm{SF} 00515$. all styles of magnets and are given in Table 1 for a 1 Tev machine. The temperature stability, harmonics, and field accuracy do not pose a problem based on the experience from the Fermilab Recycler ${ }^{2}$. To achieve the required pole tip field rare earth permanent magnets are required. Samarium Cobalt $\left(\mathrm{Sm}_{2} \mathrm{Co}_{17}\right)$ was chosen for its high residual $\mathrm{B}$ field $(\mathrm{Br})$ and small temperature variation of the field.

Table 1: Magnet Requirements for a 1TeV NLC

\begin{tabular}{|l|l|}
\hline Item & Value \\
\hline Aperture & $12.7 \mathrm{~mm}$ \\
\hline Quantity Length & $288 \quad 324 \mathrm{~mm}$ \\
& $399 \quad 432 \mathrm{~mm}$ \\
& $576 \quad 965 \mathrm{~mm}$ \\
\hline Pole tip field & 0.62 Tesla for $324 \mathrm{~mm}$ \\
& 0.80 Tesla for other \\
\hline Adjustment & +0 to $-20 \%$ \\
\hline Temperature stability & $0.5 \%$ at $25 \pm 1^{\circ} \mathrm{C}$ \\
\hline Sextupole & $\mathrm{b}_{3} / \mathrm{b}_{2}<0.02$ at $\mathrm{r}=5 \mathrm{~mm}$ \\
\hline Field accuracy & $\pm 0.5 \%$ at any field \\
\hline Center location & To Fiducial $\pm 0.1 \mathrm{~mm}$ \\
\hline Center stability & \pm 0.001 mm over range \\
& of adjustment \\
\hline
\end{tabular}

The center stability requirement of $\pm 0.001 \mathrm{~mm}$ is driven by the Beam Based Alignment (BBA) process for these quads. When a beam position monitor detects movement of the beam the position of the related quadrupole will be adjusted to bring the beam back on the correct trajectory. The BPM to quadrupole center calibration process requires that the quad strength be lowered by $20 \%$ over several seconds during which change the magnetic center must not shift by more than 1 micron. This calibration will be done monthly.

Four different styles of quadrupole were designed and built. These are called the corner tuner, wedge tuner, sliding shunt, and the rotating quad. This paper briefly describes each style and the results of testing the magnetic center stability of each one's prototype using a stretched wire measurement system. The magnets were modeled using PANDIRA and TOSCA. 


\section{CORNER TUNER}

The corner tuner magnet was similar in design to the Fermilab Recycler quadrupoles ${ }^{3}$. A C1008 low carbon steel pole with $\mathrm{Sm}_{2} \mathrm{Co}_{17}$ magnet bricks behind the poles generated the field (Figure 1). In the corners cylindrical $\mathrm{Sm}_{2} \mathrm{Co}_{17}$ magnets, $19 \mathrm{~mm}$ in diameter, magnetized across the diameter were rotated to change the strength of the gradient. The pole tip shape was a hyperbola with an inscribed radius of $12.7 \mathrm{~mm}$. Flux returns of C1008 steel surrounded the poles and magnets with outside dimensions of 158 by $158 \mathrm{~mm}$. Figure 2 shows the magnetic center as a function of angle for 360 degrees rotation of the tuning rods. The magnetic center moves by over 100 microns for a $20 \%$ change in gradient.

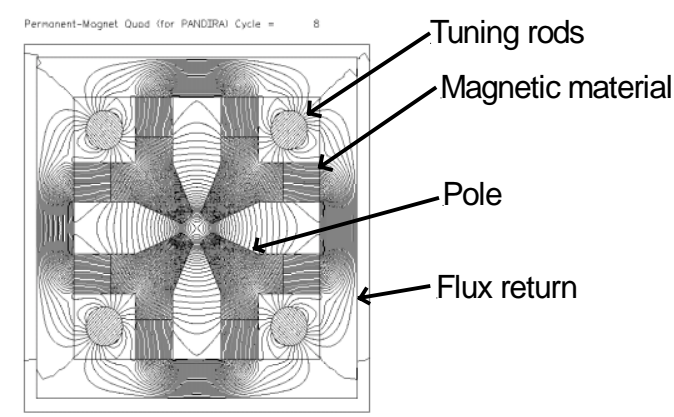

Figure 1: Corner Tuner

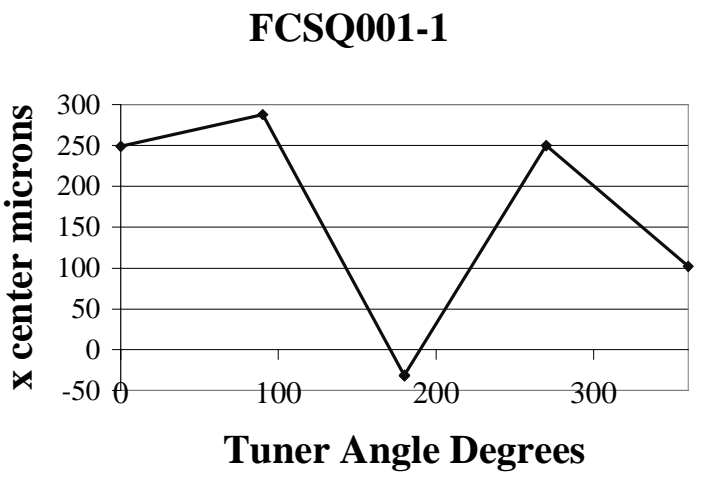

Figure 2: X Center vs. Tuning Rod Angle

\section{WEDGE TUNER}

The wedge tuner uses 2 sets of fixed $\mathrm{Sm}_{2} \mathrm{Co}_{17}$ bricks, one behind the poles and the other wedged between the poles, (Figure 3) hence the name. Also behind the poles were tuning rods as in the corner tuner. The poles were heat treated C1008 low carbon steel cut on an Electric Discharge Machine (EDM). The coordinates used in the PANDIRA model were directly fed into the EDM machine to produce the shape of the poles. To prevent the strength of the magnet from varying more than $0.01 \%$ per ${ }^{\circ} \mathrm{C}$ high nickel steel ${ }^{4}$ was inserted between the poles and flux returns. The strength of the first prototype wedge tuner was $20 \%$ lower than the designed based on PANDIRA. This is due to the difficulty of representing the effect of the temperature compensator material in the model,

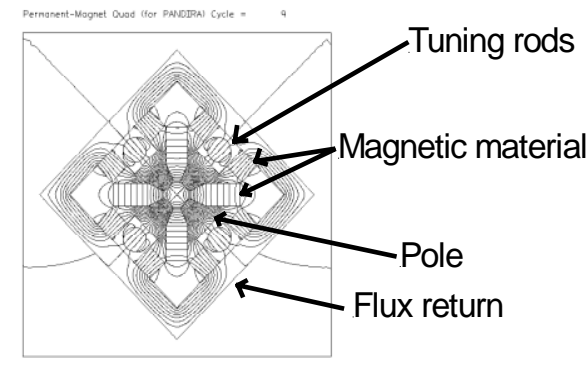

Figure 3: Wedge Quadrupole

differences in the $\mathrm{Br}$ and $\mathrm{Hc}$ of the magnetic material actually used and the values used in PANDIRA, and some loss of field from the ends. A new model that expanded the volume of magnetic material by $30 \%$ attained the required field. Figure 4 shows the center position in microns versus the angle of the tuning rods for different tuning rod arrangements. A center movement of 20 microns is observed. The different curves indicate too great an imbalance among the strengths of the tuning rods.

\section{FWSQ002 rod swap}

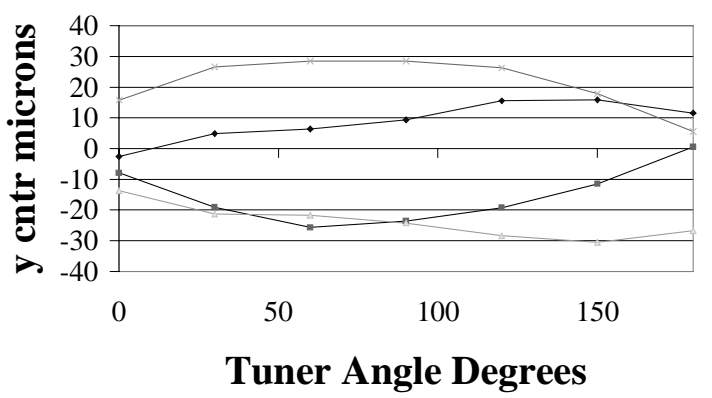

Figure 4: Y Center vs Tuning Rod Angle

\section{SHUNT TUNER}

The third design used a sliding shunt to adjust the field in the gap. The magnetic material was $\mathrm{Sm}_{1} \mathrm{Co}_{5}$ The outer flux return slides longitudinally along the body of the magnet. The poles have high and low portions (Figure 5) that allow flux to enter the steel return or the gap to change the gradient. The maximum integrated field is 25.9 Tesla and the minimum is 21.8 Tesla for a total change of $16 \%$ in the gradient. The advantage of this style is that there is only one drive needed to change the gradient. A larger magnet is under construction that will have the required $20 \%$ range in gradient. 


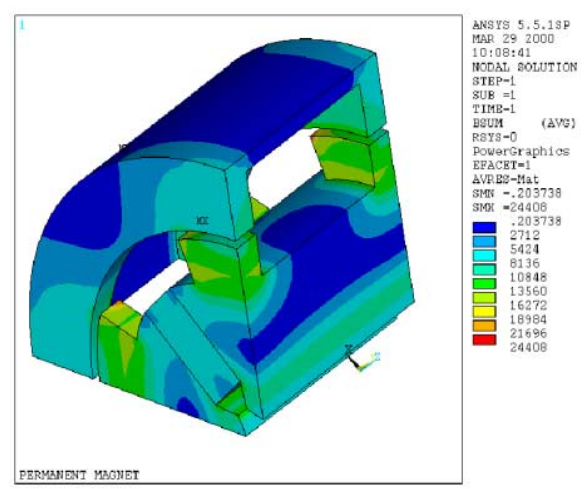

Figure 5: Sliding Shunt

Figure 6 shows the change in y center versus field for multiple movements of the sliding shunt both forward and reverse direction. The range of center motion is on the order of 15 microns.

\section{FSSQ001}

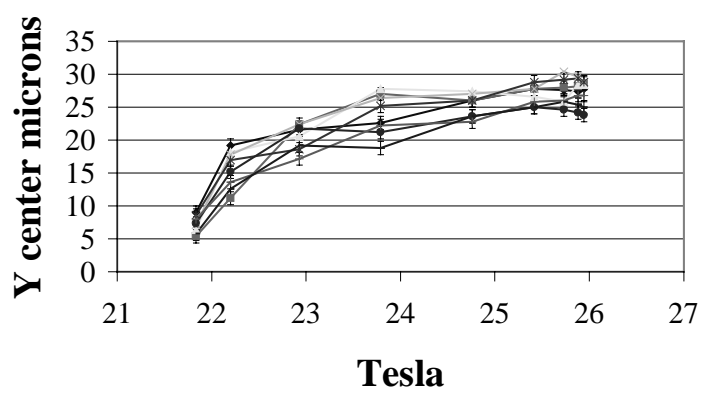

Figure 6: Ycenter vs $\int$ Gdl (y)

\section{ROTATING QUADROPOLES}

The fourth type of adjustable magnet consists of four segments. The two inner segments are counter rotating quadrupoles and the two outer segments are fixed quadrupoles. The rotating magnets provide the adjustment while the fixed quad provides the bulk of the focusing field. The rotating segments move in opposite direction to adjust the intergrated quadrupole strength. Figure 7 shows the center versus angle over the full range. The full center motion is 1 micron in $\mathrm{X}$ and 4.5 micron in $\mathrm{Y}$ for the range in gradient of 36.3 Tesla to 30.3 Tesla or $19.4 \%$.

\section{ELECTROMAGNET}

An electromagnet with the same pole shape was built at SLAC $^{5}$ and measured with a rotating coil. The integrated field varied from 27.38 Tesla to 33.22 Tesla for a current change of 120 to 150 amps. When the operating current was reached from a lessor current the center of the magnetic field remained stable to less than 1 micron.

\section{FSRQ001}

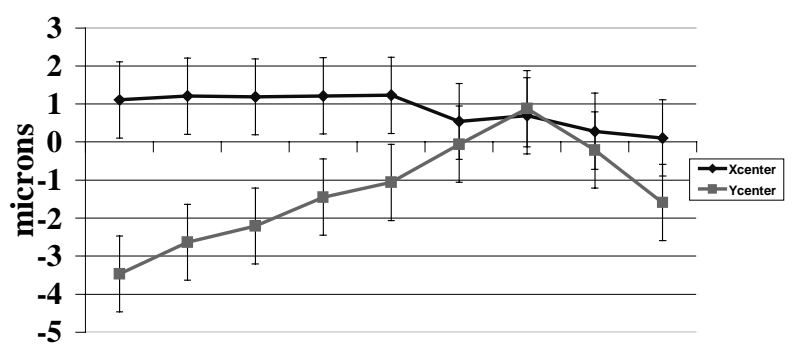

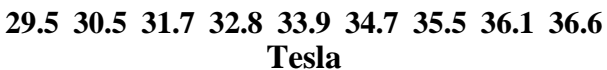

Figure 7: X Center vs $\int \mathbf{G d l}(\mathbf{x})$ Rotating quad

\section{CONCLUSION}

Four types of permanent magnet adjustable quadrupoles were built and tested. All prototypes meet the NLC requirements with the exception of magnetic center stability. The rotating quadrupoles nearly achieve the center stability specification. For all magnets further work on motor drives, bearings and balancing the magnetic material continues and should lead to center stability improvements. Table 2 gives the results for all four magnets.

Table 2

\begin{tabular}{|l|c|c|c|}
\hline & $\begin{array}{l}\text { Max grad } \\
\text { Tesla }\end{array}$ & $\begin{array}{l}\text { Min grad } \\
\text { Tesla }\end{array}$ & $\begin{array}{l}\text { Center } \\
\text { Shift } \mu\end{array}$ \\
\hline Corner & 17.5 & 14.1 & 100 \\
\hline Wedge & 23.7 & 18.4 & 20 \\
\hline Sliding shunt & 25.9 & 21.8 & 15 \\
\hline Rotating & 36.3 & 30.3 & 4.5 \\
\hline Electromagnet & 33.2 & 27.4 & 1 \\
\hline
\end{tabular}

1). Tor. O. Raubenheimer "Overview of the $X$ Band R\&D Program", submitted to this conference and "Progress in the Next Linear Collider Design", SLAC-PUB-8672, October 2000

2). Stephen D Holmes "Status of the Fermilab Main Injector and Recycler" PAC1997 Conf 97-47

3). H.D.Glass et al "Hybrid permanent magnet quadrupoles for the recycler ring at Fermilab" PAC 1997 Conf $97-337$

4). W.B. Fowler "Experiences with Procurement of Ferrite and Temperature Compensator for Permanent Magnets for Accelerators" PAC 1997 Conf 97-248

5). C.M. Spencer et al "High Reliability Prototype Quadrupole for the Next Linear Collider "submitted to Magnet Technology 17 Conference, September 2001 